\title{
Physical Layer Control for Dis-aggregated Optical Systems
}

\author{
Daniel C. Kilper \\ College of Optical Sciences \\ University of Arizona \\ Tucson, AZ USA \\ dkilper@optics.arizona.edu
}

\author{
Shengxiang Zhu \\ Electrical \& Computer Engineering \\ University of Arizona \\ Tucson, AZ, USA \\ szhu@email.arizona.edu
}

\author{
Jiakai $\mathrm{Yu}$ \\ Electrical \& Computer Engineering \\ University of Arizona \\ Tucson, AZ, USA \\ jiakaiyu@email.arizona.edu
}

\begin{abstract}
Disaggregating optical communication systems can impact physical layer control. Recent progress on multi-domain transmission control and machine-learning provide capabilities for adaptation and development of engineering rules in the field with potential benefits for disaggregated systems.
\end{abstract}

Keywords-optical system disaggregation, optical communication system, optical networks

\section{INTRODUCTION}

The introduction of alien wavelengths in optical transmission systems is a form of system disaggregation that has found widespread application. Recently, more extensive forms of disaggregation have been proposed and introduced commercially through a multisource agreement specification, which allows for sub-systems developed by different system vendors to interoperate within the same network [1-3]. Historically optical systems have been designed as end-to-end proprietary platforms that use performance margins to account for the many physical layer uncertainties and performance variations. Careful design, simulation, and lab testing of the component properties, physical layer control methods, and transmission algorithms are used to minimize performance margins [4]. Without the benefit of end-to-end control and design of the system, one can expect larger margins will be needed, which can lead to higher cost in the form of increased optical signal regeneration. However, these might be offset by a reduction in regeneration at network domain boundaries between different vendors' systems [5]. A preliminary analysis has indicated that increased disaggregation can lead to large margin erosion in longer reach systems [6].

Physical layer control is one aspect of optical systems that is proprietary to each vendor and involves the end-to-end system design. Here physical layer control refers to both the path computation element (PCE) or quality of transmission (QoT) algorithms as well as the associated optical power and switch control algorithms used to implement PCE/QoT results and maintain stable system performance and operation. Depending on the extent of disaggregation, the physical layer control may involve a range of implementations from an interoperation between proprietary control planes to a complete re-architecting of the physical layer control into a virtualized, software defined networking (SDN) based controller [7]. The latter case in fact can be thought of as a disaggregation of the physical layer controller from the optical system. Remote controls can also be augmented by autonomous local controls such as amplifier gain control and automatic channel power leveling.

New approaches to physical layer control may help to enable system disaggregation. Multi-domain PCE algorithms have been developed and used with transparent, alien wavelength, inter-domain transmission and resilience [8-12]. These can be used to better integrate systems running independent control planes, while sharing alien wavelengths. Machine learning methods [13-16] potentially can make use of field data to tighten margins or account for the diverse system implementations/characteristics. Transfer learning might be employed to translate algorithms based on lab test data or field collected data for application to new system configurations [17]. In general, greater use of field data in order to adapt system performance has the potential to reduce the reliance on lab testing and improve performance prediction, which can help to recover lost performance and reduce margins that result from disaggregation.

\section{SYSTEM DISAGGREGATION}

Dis-aggregation is a market concept that can have important ramifications on the design and performance of the respective system. Desktop computing systems, for example, began as integrated systems, but today are mostly disaggregated such that a well-informed consumer can purchase stand-alone computer components, all from different suppliers, and assemble their own system. Not only keyboard and display, but also hard drives, memory, and even the microprocessor can be purchased separately. Component pairings, however, do impact performance and require some expertise or experimentation in order to get peak performance. System integrators offer computer systems with different component options that have been tested or vetted in advance for compatibility. Consumers may also have a choice of operating system.

Today many analogous elements of this example with desk top computer disaggregation exist for optical systems. Complete reconfigurable optical add drop multiplexer (ROADM) nodes with wavelength selective switches and amplifiers are commercially available. These can be purchased as 'whiteboxes' with no controller or 'brightboxes' with controllers on board [18]. A software solution for the 
'operating system' is not currently available, although several network operating systems and SDN controllers have adopted optical component control capabilities that would make it possible to develop such a software control capability [19-20].

The openROADM multi-source agreement specifies standard ROADM interfaces and local control operations that are meant to allow for each ROADM node to be operated independently using such an SDN controller [2]. The standard is designed to avoid control signals passed directly between neighboring nodes. All control messages are passed directly to each node from an SDN controller. In general, a variety of different hierarchical control system architectures may be implemented $[3,5,18]$.

\section{OPTICAL SYSTEM DISAGgREGATION MODELS}

Optical systems can be disaggregated by dividing both hardware or software into different elements, in order to open one or both to multi-vendor supply chains. One breakdown of the different disaggregation elements is listed below and can be thought of as hierarchical (top to bottom, 1 to 3 ) layers.

Hardware Disaggregation Element Layers:

1. Wavelength ('alien wavelengths')

2. Node

3. Component

Software Disaggregation Element Layers:

1. NMS/EMS

2. Physical layer controller

Here component disaggregation refers to the major system components, including amplifiers, wavelength selective switches, optical channel monitors, mux/demux, and optical transceivers; whereas, node disaggregation takes groupings of these components that make up a node or node sub-system, such as one degree of a ROADM. Today, wavelength and network and element management systems (NMS/EMS) disaggregation are commonly supported [18]. Each of these disaggregation elements can be taken individually or separately, although the elements tend to be hierarchical in the layers shown in each list. Thus, if the components are disaggregated, then the higher (top) layer nodes and wavelengths would effectively be disaggregated. Likewise, it would be difficult to disaggregate the physical layer controller without also disaggregating the network and element management systems. There is also coupling between the software and hardware elements. While each of the software elements can be disaggregated without any hardware disaggregation (assuming the hardware is suitably specified or standardized), node and component disaggregation would require a disaggregation of all software elements.

Within each disaggregation element layer there can also be different implementation approaches. For example, for node disaggregation, the nodes can be required to meet specific requirements or standards that can tighten the resulting performance variations.

\section{Physical Layer CONTROL}

Several different aspects of physical layer control will be impacted by disaggregation. For wavelength disaggregation, challenges include untested 'alien' signals and the inability to perform end-to-end testing on the signals. It can also involve interoperation of alien wavelengths between different systems. Different transceivers may perform better at different power levels, achieve different transmission reach, or show unique sensitivity to nonlinear effects and channel cross-talk. Lab testing is often used to prove in the performance of such alien wavelengths prior to deployment. The ability to adapt channel route, spacing and power 'online' within the system in the field, based on the alien wavelength performance can potentially reduce the dependence on pre-deployment testing and tighten margins.

For the case of multiple systems of different vendor origin in the same network, sharing alien wavelengths, the additional problem exists of physical layer control plane coordination between systems. This type of scenario has been studied for multi-domain transmission in terms of coordinating PCEs for routing and wavelength assignment and for performance guarantees and restoration using optical performance monitoring [21-23].

The greater variability in transmission performance that occurs with deeper layers of disaggregation has been addressed through several directions. One approach is to provide tight specifications or standards that yield greater uniformity in the constituent elements and how they can be operated $[2,18]$. A disaggregated physical layer controller is needed in this case to operate the system. These disaggregated controllers will need to contend with the greater variability in component pairings than found in a proprietary system. Maintaining large margins, for example by constraining the size of the system, may accommodate the larger performance variability [6].

Another approach to addressing the performance variability in disaggregated systems is to introduce new monitoring and control in the field that can adapt to the greater diversity in system configuration. Machine learning in particular might be used either through reinforcement or transfer to tune the controls based on the reality in the field. Transfer learning has been used to show a QoT estimator that enables an algorithm developed in the lab to be adapted with only a few training cycles to a new system configuration. Training on a LEAF fiber based lab system was used to achieve accurate $Q$ factor predictions on a different LEAF fiber lab system and on a system with different fiber type and modulation format, suing, with only 20 new training samples [17]. Such an approach might be used when a disaggregated system is deployed in order to quickly learn the appropriate QoT model. Similarly, reinforcement learning might be used to improve the system operation and tighten margins. In a recent analysis, the use of field measured data was shown to result in dramatic reductions in the QoT prediction error for wavelength routing in a panEuropean network [16].

Using machine learning to better predict performance based on the unique system configurations can tighten margins in general, which can enable the use of disaggregation in larger systems. Recently machine learning was used to accurately predict wavelength dependent channel powers, which is the primary determinant of the optical signal to noise ratio and the impact of nonlinear optical impairments [11, 24, 25]. 


\section{CONCLUSION}

Disaggregation of optical systems can impact the requirements on performance prediction and control for optical transmission. Different layers of disaggregation were introduced and discussed in the context of the physical layer control requirements. Top layer disaggregation leads to a multidomain inter-operability problem; whereas, lower layer disaggregation can require new control architectures and new approaches to dealing with greater uncertainty in the system constituent elements and reduced end-to-end system engineering. Recent progress in multi-domain physical layer prediction and control and machine learning methods hold promise to address these challenges.

\section{ACKNOWLEDGMENT}

The authors appreciate thoughtful discussion on these topics with Weiyang Mo, Yao Li, Houman Rastegarfar, and Marco Ruffini.

\section{REFERENCES}

[1] Kreutz, Diego, et al. "Software-defined networking: A comprehensive survey." Proceedings of the IEEE 103.1 (2015): 14-76.

[2] OpenROADM. [Online]. Available: http://www.openroadm.org

[3] Li, Yao, and Kilper, Daniel C.. "Optical physical layer SDN." IEEE/OSA Journal of Optical Communications and Networking 10.1 (2018): A110-A121.

[4] Leplingard, Florence, et al., "Determination of the impact of a quality of transmission estimator margin on the dimensioning of an optical network," In Proc. Optical Fiber Communication Conference, 2008.

[5] Santos, Joao, Costa, Nelson, and Pedro, Joao, "On the impact of deploying optical transport networks using disaggregated line systems," in IEEE/OSA Journal of Optical Communications and Networking, vol. 10, no. 1, pp. A60-A68, Jan. 2018. doi: 10.1364/JOCN.10.000A60

[6] Belanger, Michel P., O'Sullivan, Maurice and Littlewood, Paul, "Margin Requirement of Disaggregating the DWDM Transport System and Its Consequence on Application Economics," 2018 Optical Fiber Communications Conference and Exposition (OFC), San Diego, CA, 2018, paper M1E.2.

[7] Thyagaturu, Akhilesh S., et al. "Software defined optical networks (SDONs): A comprehensive survey." IEEE Communications Surveys \& Tutorials 18.4 (2016): 2738-2786.

[8] Liu, Lei et. al., "Interworking between OpenFlow and PCE for dynamic wavelength path control in multi-domain WSON," in Proc. Opt. Fiber Commun. Conf. , 2012, pp. 1-3.

[9] Gonzalez de Dios, Oscar, et al., "First demonstration of multi-vendor and multi-domain EON with S-BVT and control interoperability over Pan-European testbed," in Proc. Eur. Conf. Exhib. Opt. Commun., 2015, pp. 1-3.
[10] Marconett, Dan, Liu, Lei, and Yoo, S. J. B. "Optical FlowBroker: Loadbalancing in software-defined multi-domain optical networks," in Proc. Opt. Fiber Commun. Conf. Exhib., 2014, pp. 1-3.

[11] Yoo, S. J. B. "Multi-domain cognitive optical software defined networks with market-driven brokers," in Proc. Eur. Conf. Exhib. Opt. Commun., 2014, pp. 1-3.

[12] Li, Yao, et al. "tSDX: enabling impairment-aware all-optical interdomain exchange." Journal of Lightwave Technology 36.1 (2018): 142154.

[13] Barletta, Luca, Giusti, Alessandro, Rottondi, Cristina and Tornatore, Massimo, "QoT estimation for unestablished lighpaths using machine learning," 2017 Optical Fiber Communications Conference and Exhibition (OFC), Los Angeles, CA, 2017, pp. 1-3.

[14] Gutterman, Craig L., et al. "Neural network based wavelength assignment in optical switching." Proceedings of the Workshop on Big Data Analytics and Machine Learning for Data Communication Networks. ACM, 2017.

[15] Aladin, Sandra and Tremblay, Christine, "Cognitive Tool for Estimating the QoT of New Lightpaths," 2018 Optical Fiber Communications Conference and Exposition (OFC), San Diego, CA, 2018, pp. 1-3.

[16] Seve, Emmanuel, et. al., "Learning process for reducing uncertainties on network parameters and design margins," in IEEE/OSA Journal of Optical Communications and Networking, vol. 10, no. 2, pp. A298A306, Feb. 2018. doi: 10.1364/JOCN.10.00A298

[17] Mo, Weiyang, et al. "ANN-based transfer learning for QoT prediction in real-time mixed line-rate systems." 2018 Optical Fiber Communications Conference and Exposition (OFC). IEEE, 2018.

[18] Riccardi, Emilio, et. al., "An Operator view on the Introduction of White Boxes into Optical Networks," in Journal of Lightwave Technology, vol. 36, no. 15, pp. 3062-3072, 1 Aug.1, 2018. doi: 10.1109/JLT.2018.2815266

[19] Berde, Pankaj, et al. "ONOS: towards an open, distributed SDN OS." Proceedings of the third workshop on Hot topics in software defined networking. ACM, 2014.

[20] Medved, Jan, et al. "Opendaylight: Towards a model-driven sdn controller architecture." 2014 IEEE 15th International Symposium on. IEEE, 2014.

[21] Lopez, Victor, et al. "Demonstration of SDN orchestration in optical multi-vendor scenarios." Optical Fiber Communications Conference and Exhibition (OFC), 2015. IEEE, 2015.

[22] Giorgetti, Alessio, et al. "Dynamic restoration with GMPLS and SDN control plane in elastic optical networks." Journal of Optical Communications and Networking 7.2 (2015): A174-A182.

[23] Casellas, Ramon, et al. "SDN orchestration of OpenFlow and GMPLS flexi-grid networks with a stateful hierarchical PCE." Journal of Optical Communications and Networking 7.1 (2015): A106-A117.

[24] Mo, Weiyang, et al. "Deep-Neural-Network-Based Wavelength Selection and Switching in ROADM Systems." Journal of Optical Communications and Networking 10.10 (2018): D1-D11.

[25] Zhu, Shengxiang, et al. "Machine Learning Based Prediction of ErbiumDoped Fiber WDM Line Amplifier Gain Spectra." European Conference On Optical Communication, 2018, paper Mo3E.6. 